\title{
Investigation of the Nuclear Structure for Some p-Shell Nuclei by Harmonic Oscillator and Woods-Saxon Potentials
}

\author{
Ahmed N. Abdullah \\ Department of Physics, College of Science, University of Baghdad, Baghdad-Iraq. \\ Corresponding Author: Ahmednajim1979@yahoo.com
}

\begin{abstract}
The single particle radial wave functions of harmonic oscillator potential and Woods-Saxon potential have been used to calculate the charge density distributions, charge form factor and the matter and charge root mean square (rms) radii for some odd-A $1 \mathrm{p}$ shell nuclei, namely ${ }^{9} \mathrm{Be},{ }^{11} \mathrm{~B}$, ${ }^{13} \mathrm{C}$ and ${ }^{15} \mathrm{~N}$ nuclei. The calculated results are compared with the measured data. Calculations show that both the Wood-Saxson (WS) and harmonic oscillator (HO) potentials are capable of providing theoretical predictions on the structure of $\mathrm{p}$-shell nuclei and be in a satisfactory description with those of experimental data. The quadrupole form factors contribution for ${ }^{9} \mathrm{Be}$ and ${ }^{11} \mathrm{~B}$ nuclei are carried out using undeformed p-shell model and give a well accordance with the measured results.

[DOI: 10.22401/JNUS.20.2.06]
\end{abstract}

Keywords: Harmonic oscillator potential, Woods-Saxon potential, charge density distributions, quadrupole form factors, p-shell Nuclei.

PACS Nos.: 21.10.Ft, 25.30.Bf

\section{Introduction}

The charge density distribution has been well studied experimentally over a wide range of nuclei because it is one of the many most important quantities in the nuclear structure [1]. The important information about the nuclear structure is obtained from the high energy electron scattering by the nuclei. At high energy, in the range of $100 \mathrm{MeV}$ and more, the electron represents a best probe to study the nuclear structure because with these energies the de Broglie wavelength will be in the range of the spatial extension of the target nucleus [2]. We can distinguish two different types of the electron scattering: the first type is called elastic electron scattering where the nucleus is left on its ground state. The second is inelastic electron scattering where the nucleus is left in its different excited states [3].

Gibson [4] has been study the ground state of the ${ }^{4} \mathrm{He}$ nucleus using a single-particle phenomenological model. Wave functions were generated from a potential whose parameters are chosen to reproduce the correct neutron separation energy. Ridha [5] has been used the single-particle radial wave functions of Woods-Saxon potential and harmonicoscillator potential to study the nuclear charge density distributions, form factors and corresponding proton, charge, neutron, and matter root mean square radii for stable ${ }^{4} \mathrm{He}$, ${ }^{12} \mathrm{C}$, and ${ }^{16} \mathrm{O}$ nuclei. The obtained results have been compared with experimental data. Lojewski [6] has been evaluated the meansquare charge radii of even-even nuclei using the realistic single-particle Woods-Saxon potential and compared the results with experimental data and theoretical values obtained with the single-particle Nilsson potential. Gamba [7] determined the parameters of a Woods-Saxon potential well for ten $\mathrm{p}$-shell nuclei by fitting the electron scattering form factors and single-particle binding energies.

The aim of the present investigation is the analysis of the charge and matter root mean square (rms) radii, charge density distributions and charge form factors for some $\mathrm{p}$-shell nuclei like ${ }^{9} \mathrm{Be},{ }^{11} \mathrm{~B},{ }^{13} \mathrm{C}$ and ${ }^{15} \mathrm{~N}$ nuclei calculated with the radial wave function of Woods-Saxon and harmonic oscillator potentials and compared the results with experimental data.

\section{Theory}

The Woods-Saxon potential is the sum of a spin-independent central potential, a spin-orbit potential, and the Coulomb potential $[7,8,9]$ :

$V(r)=V_{0}(r)+V_{s o}(r) \vec{\ell} \cdot \vec{s}+V_{c}(r)$, 
where $V_{0}(r)$ is the spin-independent central potential:

$V_{0}(r)=V_{0} f_{0}(r)$,

with a Fermi shape

$f_{0}(r)=\frac{1}{1+\left[e^{\left(r-R_{0}\right) / a_{0}}\right]}$

$\mathrm{V}_{\text {so }}(\mathrm{r})$ is the spin-orbit potential:

$V_{s o}(r)=V_{s o} \frac{1}{r} \frac{d f_{s o}(r)}{d r}$,

with

$f_{\text {so }}(r)=\frac{1}{1+\left[e^{\left(r-R_{s o}\right) / a_{s o}}\right]}$

and $V_{c}(r)$ is the Coulomb potential for protons based upon the Coulomb potential for

a sphere of radius $R_{\mathrm{c}}$ :

$V_{c}(r)=\frac{Z e^{2}}{r} \quad$ for $\quad r \geq R_{c}$

$V_{c}(r)=\frac{Z e^{2}}{R_{c}}\left[\frac{3}{2}-\frac{r^{2}}{2 R_{c}^{2}}\right]$ for $r \leq R_{c}$

The radii $R_{0}, R_{\text {so }}$ and $R_{\mathrm{c}}$ are usually expressed as:

$R_{i}=r_{i} A^{1 / 3}$

The point density distributions of protons, neutrons and matter can be written, respectively as [1]:

$\rho_{g}(r)=\frac{1}{4 \pi} \sum_{n \ell j} X_{g}^{n \ell j}\left|R_{n \ell j}\right|^{2}, g=p, n, m$

where $X_{g}^{n \ell j}$ represents the number of protons, neutrons or nucleons in the $n \ell j$ subshell.

The normalization condition of the above ground state densities is given by [10]:

$g=4 \pi \int_{0}^{\infty} \rho_{g}(r) r^{2} d r$

where $\rho_{g}(r)$ represents one of the following densities $\left[\rho_{p}(r), \rho_{n}(r)\right.$ or $\left.\rho_{m}(r)\right]$ and $g$ corresponds to the number of nucleon in each case. The rms radii of corresponding above densities are given by [10]:

$$
\left\langle r^{2}\right\rangle_{g}^{1 / 2}=\frac{4 \pi}{g} \int_{0}^{\infty} \rho_{g}(r) r^{4} d r
$$

The total charge form factor squared for unpolarized electrons and target is given by $[11,12]$ :

$\left|F_{c h}(q)\right|^{2}=\left|F_{0}(q)\right|^{2}+\left|F_{2}(q)\right|^{2}$

The elastic charge form factor, $F_{0}(q)$, is calculated by the Fourier transform of the ground state charge density distribution [10], i.e.

$F_{0}(q)=\frac{4 \pi}{Z} \int_{0}^{\infty} \rho_{0 c h}(r) j_{0}(q r) r^{2} d r$

where $j_{0}(q r)$ is the spherical Bessel function of order zero and $q$ is the momentum transfer from the incident electron to the target nucleus.

The normalization of the ground state charge density distributions, $\rho_{0 c h}$, is given by [11]:

$Z=4 \pi \int_{0}^{\infty} \rho_{0 c h}(r) r^{2} d r$

The quadrupole form factor, $F_{2}(q)$, is obtained by the undeformed $\mathrm{p}$-shell model as $[11,12]$ :

$F_{2}(q)=\frac{\left\langle r^{2}\right\rangle}{Q}\left(\frac{4}{5 P_{J}}\right)^{1 / 2} \int \rho_{2 c h}(r) j_{2}(q r) r^{2} d r$

where $j_{2}(q r)$ is the second order of the spherical Bessel functions, $Q$ is the quadrupole moment and $P_{J}$ is a quadrupole projection factor given as [12]:

$P_{J}=\frac{J(2 J-1)}{(J+1)(2 J+3)}$

\section{Results and Discussion}

The single particle radial wave functions of harmonic oscillator potential and WoodsSaxon potential have been used to calculate the charge density distributions, charge form factor and the matter and charge root mean square radii for ${ }^{9} \mathrm{Be},{ }^{11} \mathrm{~B},{ }^{13} \mathrm{C}$ and ${ }^{15} \mathrm{~N}$ nuclei. The Woods-Saxon potential is used to obtain the single-particle energies and single- particle radial wave functions for the bound states with quantum numbers $n, \ell$ and $\mathrm{j}$. The WoodsSaxon parameters $V_{0}, V_{s o}, r_{0}, r_{s o}, a_{0}, a_{s o}$ and $r_{c}$ employed in the present calculations for ${ }^{9} \mathrm{Be}$, ${ }^{11} \mathrm{~B},{ }^{13} \mathrm{C}$ and ${ }^{15} \mathrm{~N}$ nuclei are listed in Table (1). 
Table (1)

The Woods-Saxon parameters employed in the present calculations for ${ }^{9} \mathrm{Be},{ }^{11} \mathrm{~B},{ }^{13} \mathrm{C}$ and ${ }^{15} \mathrm{~N}$ nuclei.

\begin{tabular}{|c||c||c||c|c|c||c|c||}
\hline \hline Nuclei & $\boldsymbol{V}_{\mathbf{0}}(\mathbf{M e V})$ & $\boldsymbol{V}_{\boldsymbol{s o}} \mathbf{( M e V )}$ & $\boldsymbol{a}_{\mathbf{0}}(\mathbf{f m})$ & $\boldsymbol{a}_{\boldsymbol{s o}}(\mathbf{f m})$ & $\boldsymbol{r}_{\mathbf{0}}(\mathbf{f m})$ & $\boldsymbol{r}_{\boldsymbol{s o}}(\mathbf{f m})$ & $\boldsymbol{r}_{\boldsymbol{c}}(\mathbf{f m})$ \\
\hline \hline${ }^{9} \mathrm{Be}$ & 41.998 & 6 & 0.534 & 0.534 & 1.349 & 1.349 & 1.494 \\
\hline \hline${ }^{11} \mathrm{~B}$ & 63.392 & 6 & 0.772 & 0.772 & 1.295 & 1.295 & 1.462 \\
\hline${ }^{13} \mathrm{C}$ & 70.786 & 6 & 0.895 & 0.895 & 1.269 & 1.269 & 1.442 \\
\hline${ }^{15} \mathrm{~N}$ & 54.352 & 6 & 0.599 & 0.599 & 1.315 & 1.315 & 1.421 \\
\hline
\end{tabular}

The calculated matter and charge rms radii for ${ }^{9} \mathrm{Be},{ }^{11} \mathrm{~B},{ }^{13} \mathrm{C}$ and ${ }^{15} \mathrm{~N}$ nuclei along with the experimental data $[13,14,15]$ are shown in Table (2). According to these results, the calculated matter and charge rms radii for interested nuclei using both of $\mathrm{HO}$ and WS potentials are in reasonable agreement with experimental values within quoted error. The calculated single-particle energies for the investigated nuclei along with those results of the Ref. [16] are tabulated in Table-3. It is clear from this table that the obtained values of the single-particle energies are in excellent agreement with the results of the Ref. [16].

Table (2)

The calculated matter and charge rms radii for ${ }^{9} \mathrm{Be},{ }^{11} \mathrm{~B},{ }^{13} \mathrm{C}$ and ${ }^{15} \mathrm{~N}$ nuclei along with the experimental data.

\begin{tabular}{|c|c|c|c|c|c|c|}
\hline \multirow[t]{2}{*}{ Nuclei } & \multicolumn{2}{|c|}{$\begin{array}{c}\left\langle r^{2}\right\rangle_{c a l}^{1 / 2} \text { matter } \\
(\mathrm{fm})\end{array}$} & \multirow{2}{*}{$\begin{array}{c}\left\langle r^{2}\right\rangle_{\exp }^{1 / 2}(\mathrm{fm}) \\
{[13]}\end{array}$} & \multicolumn{2}{|c|}{$\left\langle r^{2}\right\rangle_{c a l}^{1 / 2}$ charge (fm) } & \multirow{2}{*}{$\begin{array}{c}\left\langle r^{2}\right\rangle_{\exp }^{1 / 2}(\mathrm{fm}) \\
{[14,15]}\end{array}$} \\
\hline & HO & WS & & HO & WS & \\
\hline${ }^{9} \mathrm{Be}$ & 2.51 & 2.68 & $2.53 \pm 0.07$ & 2.5 & 2.68 & $2.52 \pm 0.012$ \\
\hline${ }^{11} \mathrm{~B}$ & 2.60 & 2.44 & $2.6 \pm 0.09$ & 2.40 & 2.49 & $2.40 \pm 0.03$ \\
\hline${ }^{13} \mathrm{C}$ & 2.40 & 2.46 & $2.42 \pm 0.24$ & 2.46 & 2.53 & $2.46 \pm 0.003$ \\
\hline${ }^{15} \mathrm{~N}$ & 2.54 & 2.56 & $2.42 \pm 0.1$ & 2.61 & 2.64 & $2.61 \pm 0.09$ \\
\hline
\end{tabular}

Table (3)

The calculated single-particle energies for ${ }^{9} \mathrm{Be},{ }^{11} \mathrm{~B},{ }^{13} \mathrm{C}$ and ${ }^{15} \mathrm{~N}$ nuclei along with results of the Ref. [16].

\begin{tabular}{|c||c|c|c||c||c||}
\hline \multirow{2}{*}{ Nuclei } & \multirow{2}{*}{$\boldsymbol{n}_{\boldsymbol{j}}$} & \multicolumn{2}{|c|}{ Proton } & \multicolumn{2}{c||}{ Neutron } \\
\cline { 3 - 6 } & & $\mathbf{E}_{\mathrm{cal}}(\mathbf{M e V})$ & $\mathbf{E}(\mathbf{M e V})[\mathbf{1 6}]$ & $\mathbf{E}_{\text {cal }}(\mathbf{M e V})$ & $\mathbf{E}(\mathbf{M e V})[\mathbf{1 6}]$ \\
\hline \hline \multirow{2}{*}{${ }^{9} \mathrm{Be}$} & $1 \mathrm{~s}_{1 / 2}$ & 18.498 & 18.498 & 20.275 & 20.275 \\
\cline { 2 - 6 } & $1 \mathrm{p}_{3 / 2}$ & 4.971 & 4.971 & 6.499 & 6.499 \\
\hline \hline \multirow{2}{*}{${ }^{11} \mathrm{~B}$} & $1 \mathrm{~s}_{1 / 2}$ & 32.762 & 32.762 & 35.108 & 35.108 \\
\cline { 2 - 6 } & $1 \mathrm{p}_{3 / 2}$ & 16.862 & 16.862 & 18.974 & 18.974 \\
\hline \multirow{2}{*}{${ }^{13} \mathrm{C}$} & $1 \mathrm{~s}_{1 / 2}$ & 37.827 & 37.827 & 40.669 & 40.669 \\
\cline { 2 - 6 } & $1 \mathrm{p}_{3 / 2}$ & 21.912 & 21.912 & 24.496 & 24.496 \\
\hline \multirow{3}{*}{${ }^{15} \mathrm{~N}$} & $1 \mathrm{~s}_{1 / 2}$ & 31.060 & 31.060 & 34.332 & 34.332 \\
\cline { 2 - 6 } & $1 \mathrm{p}_{3 / 2}$ & 17.257 & 17.257 & 20.262 & 20.262 \\
\cline { 2 - 6 } & $1 \mathrm{p}_{1 / 2}$ & 13.827 & 13.827 & 16.833 & 16.833 \\
\hline
\end{tabular}


In Figs. 1(a) to 1(d), the calculated CDD's (in $\mathrm{fm}^{-3}$ ) of the ground state are plotted versus $r$ (in $\mathrm{fm}$ ) for ${ }^{9} \mathrm{Be},{ }^{11} \mathrm{~B},{ }^{13} \mathrm{C}$ and ${ }^{15} \mathrm{~N}$ nuclei, respectively. The red and blue distributions are the calculated CDD's using harmonic oscillator (HO) and Wood-Saxson (WS) potentials, respectively whereas the dotted symbols are the experimental data $[14,17,18,19]$. It is evident from Figs. 1(a), 1(b) and 1(d) which correspond to the ${ }^{9} \mathrm{Be},{ }^{11} \mathrm{~B}$ and ${ }^{15} \mathrm{~N}$ nuclei, respectively that both calculations of red and blue distributions are in excellent accordance with the experimental data throughout all values of $r$.
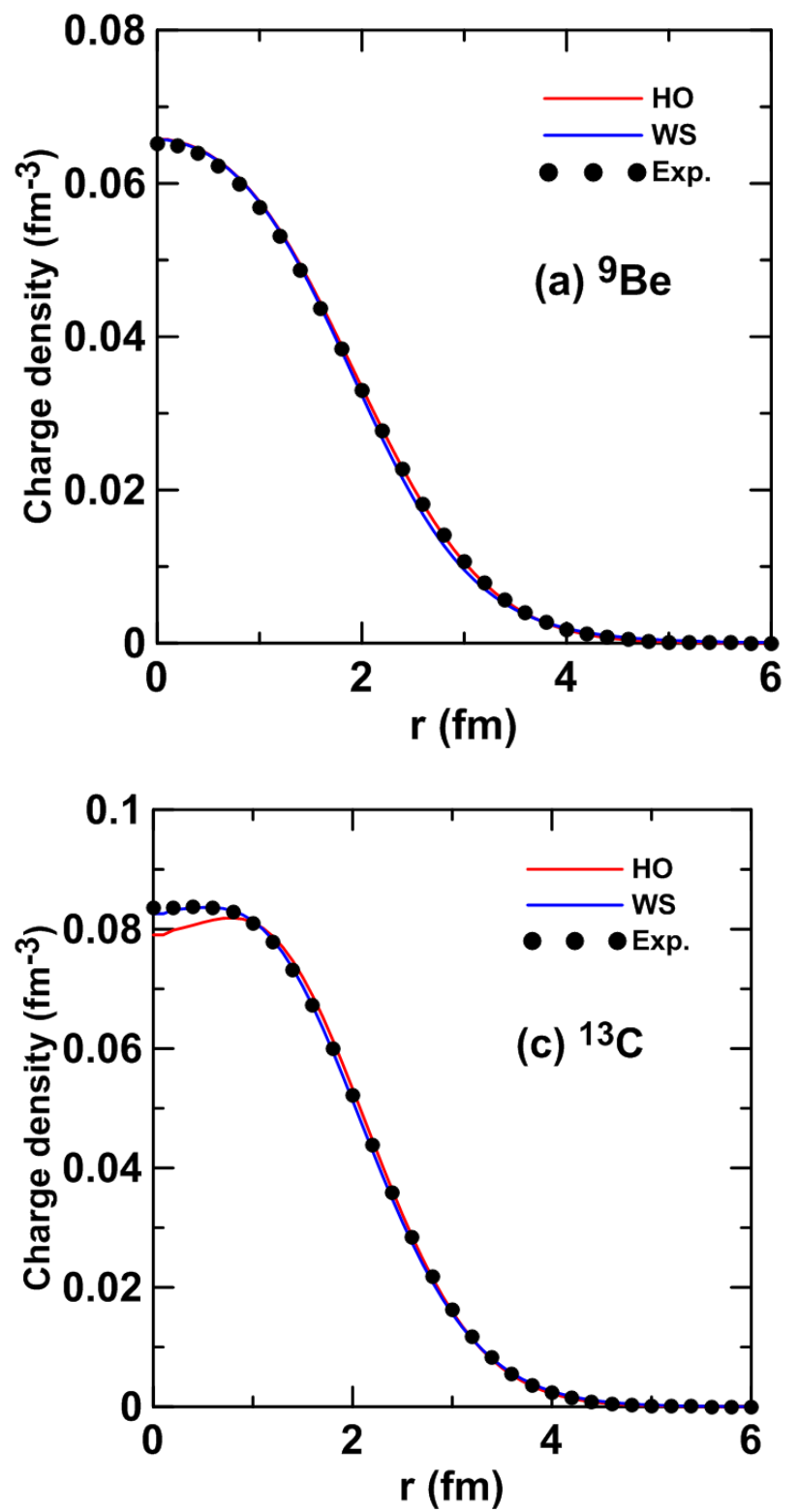

Fig.1(c) shows remarkable agreement between the blue curve and the experimental data of ${ }^{13} \mathrm{C}$ nucleus throughout the whole range of $r$. This figure also explores that the red curve underestimate slightly the experimental data at the central region while beyond this region it agree with the data very well.
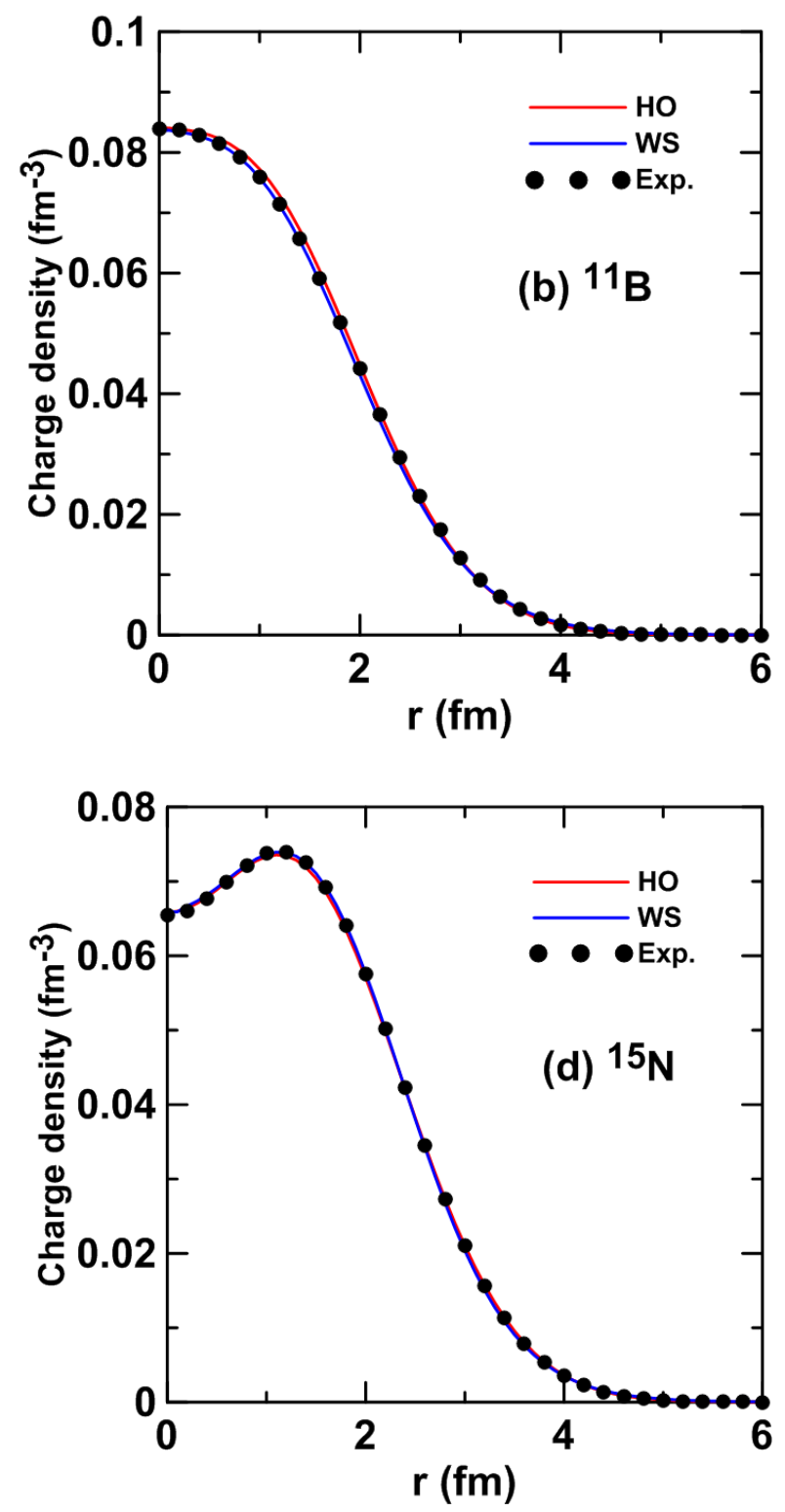

Fig.(1): The calculated charge density distributions for ${ }^{9} \mathrm{Be},{ }^{11} \mathrm{~B},{ }^{13} \mathrm{C}$ and ${ }^{15} \mathrm{~N}$ nuclei. The dotted symbols are the experimental data taken from Refs. [17], [14], [18] and [19] for ${ }^{9} \mathrm{Be},{ }^{11} \mathrm{~B},{ }^{13} \mathrm{C}$ and ${ }^{15} \mathrm{~N}$ nuclei, respectively. 
Fig.(2) exemplifies the calculated form factors obtained by the WS potential [Figs. 2(a) and 2(c)] and HO potential [Figs. 2(b) and 2(d)], where the contribution of the quadrupole form factor $\left|F_{2}(\mathrm{q})\right|^{2}$ is taking into account using the undeformed p- shell model as given in Eq. (15). The top and bottom panels correspond to ${ }^{9} \mathrm{Be}$ and ${ }^{11} \mathrm{~B}$ nuclei, respectively. For comparison the measured data [11,20,21] (denoted by rhombs and dotted symbols) are also displayed in these figures. The monopole $\left|F_{0}(\mathrm{q})\right|^{2}$ and quadrupole $\left|F_{2}(\mathrm{q})\right|^{2}$ form factors contributions are shown as dash-dotted and dashed curves, respectively, while the total contribution $|F(\mathrm{q})|^{2}$, which is obtained as the
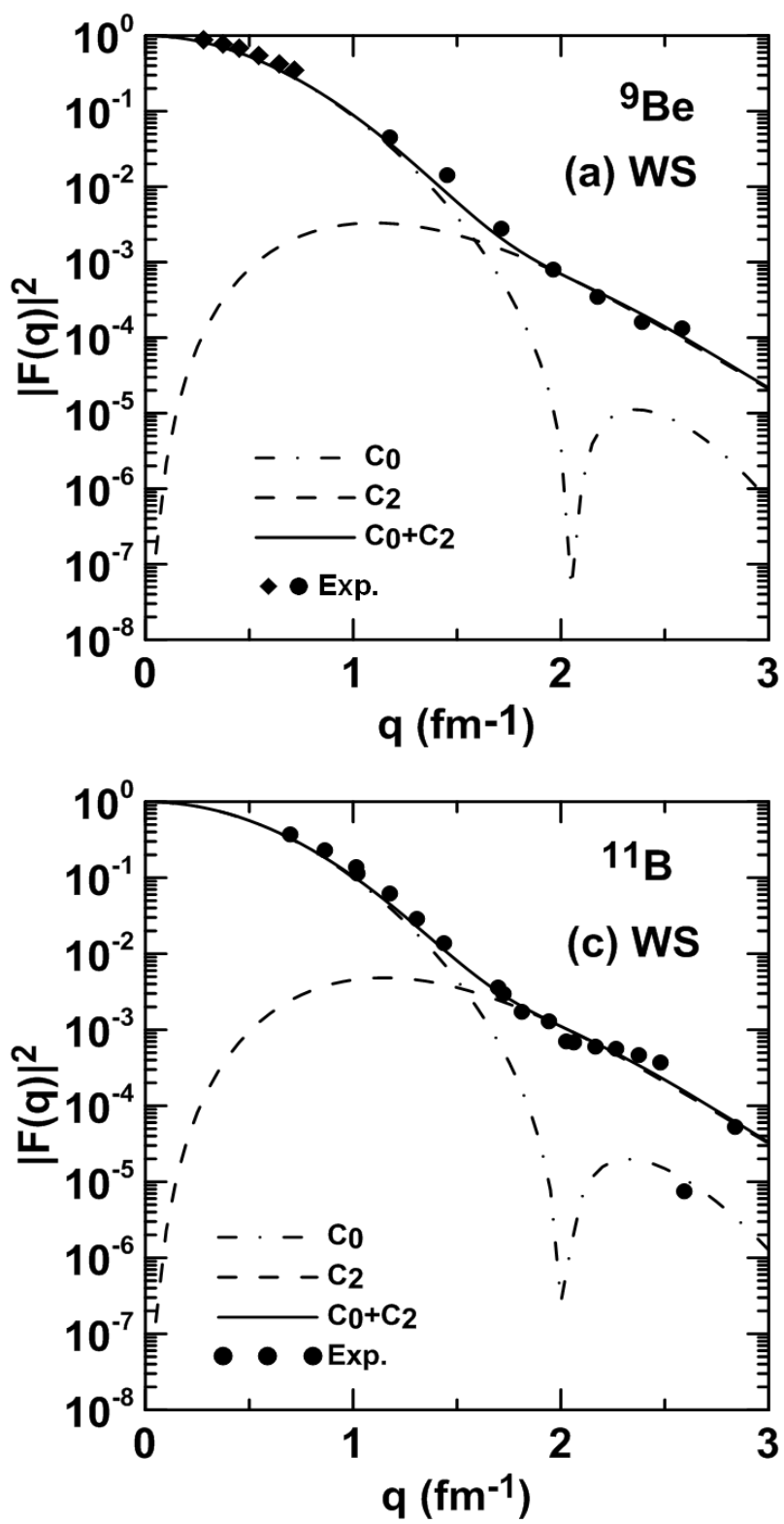

sum of $\left|F_{0}(\mathrm{q})\right|^{2}$ and $\left|F_{2}(\mathrm{q})\right|^{2}$, is represent by solid curves. It is clear from these figures that the calculated $\left|F_{0}(\mathrm{q})\right|^{2}$ for ${ }^{9} \mathrm{Be}$ and ${ }^{11} \mathrm{~B}$ nuclei are unable to give a satisfactory description with the measured data for the region of momentum transfer $\mathrm{q}>1.45 \mathrm{fm}^{-1}$. Including the contribution of $\left|F_{2}(\mathrm{q})\right|^{2}$ into our calculations improves the results and gives us a satisfactory description for all regions of momentum transfer $\mathrm{q}$ as seen in the solid distributions of these figures.
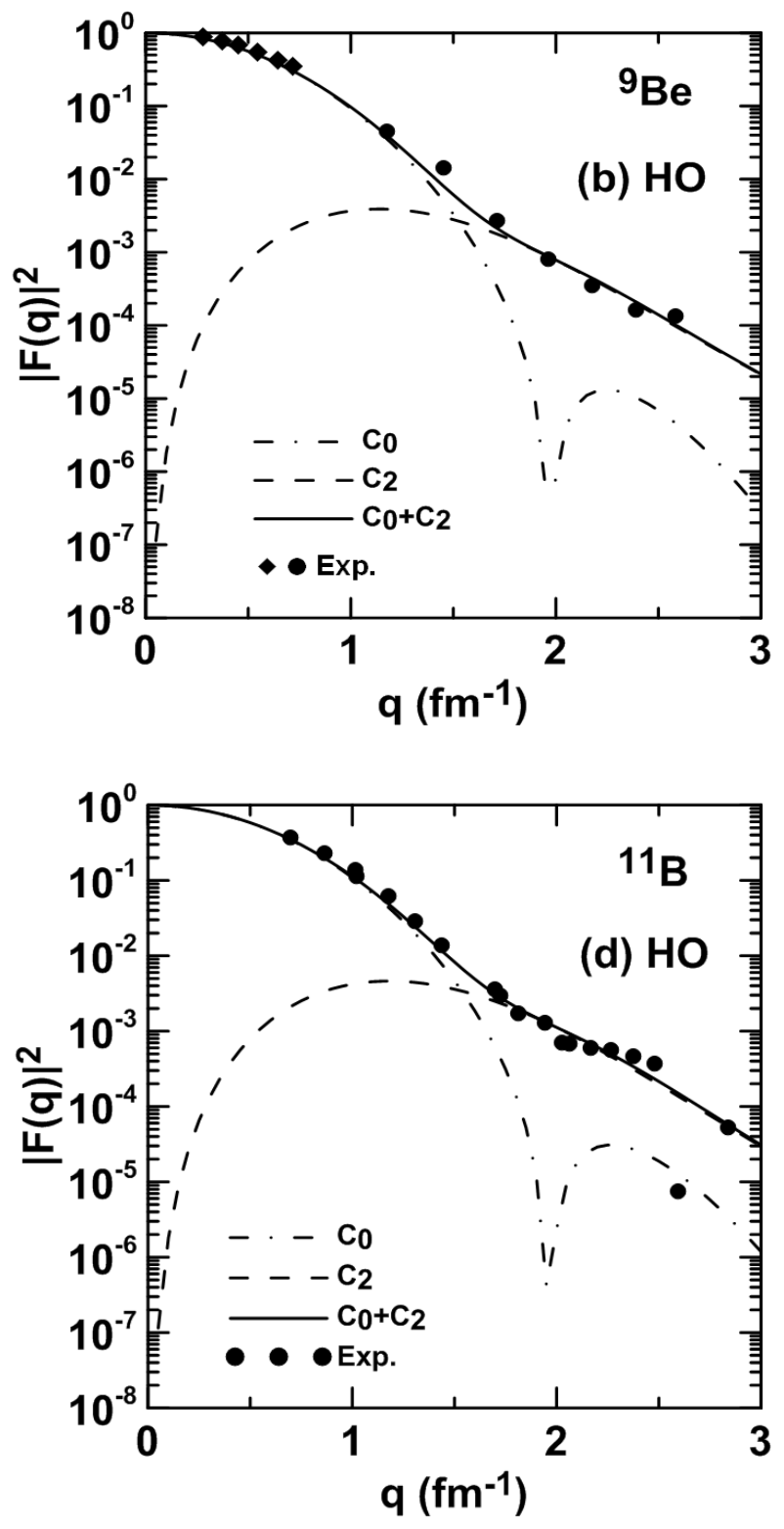

Fig. (2): The calculated form factors obtained by the WS and HO potentials for ${ }^{9} \mathrm{Be}$ and ${ }^{11} \mathrm{~B}$ nuclei. The measured results are taken from Ref. [20] (rhombs) and Ref. [21] (dotted symbols) for ${ }^{9}$ Be and taken from Ref. [11] for ${ }^{11} B$. 
Figs. 3(a) and 3(b) demonstrate the comparison between the calculated form factors of ${ }^{13} \mathrm{C}$ and ${ }^{15} \mathrm{~N}$ nuclei and experimental ones (dotted symbols) [19,22]. The solid and dashed curves correspond to the evaluated monopole form factors using harmonic oscillator (HO) and Wood-Saxson (WS) potentials, respectively. It is obvious from these figures that the obtained results of both the solid and dashed curves are coinciding with the experimental data up to $\mathrm{q} \approx 2 \mathrm{fm}^{-1}$ and they are underestimate slightly these data at higher momentum transfer. Furthermore, it

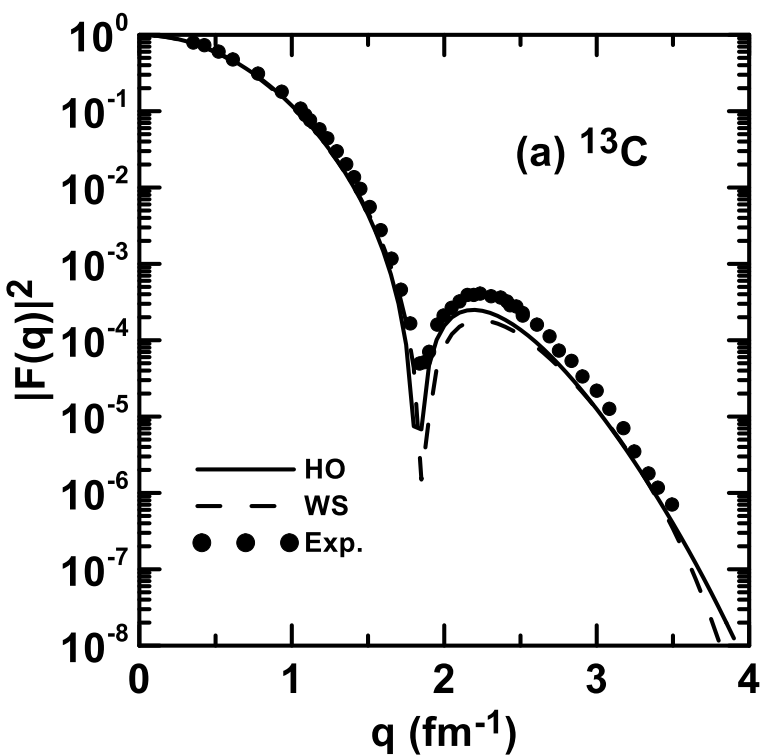

is so clear that the location of the observed diffraction minimum is reproduced in the correct place by both the dashed and solid curves. In addition, it is noted from these figures that both of the dashed and solid curves are in coincidence with each other for the region of momentum transfer $\mathrm{q} \leq 1.8 \mathrm{fm}^{-1}$. It is concluded from figures 2 and 3 that the calculated form factors with both WS and $\mathrm{HO}$ potentials of nuclei under investigation are in a good agreement with the experimental data.

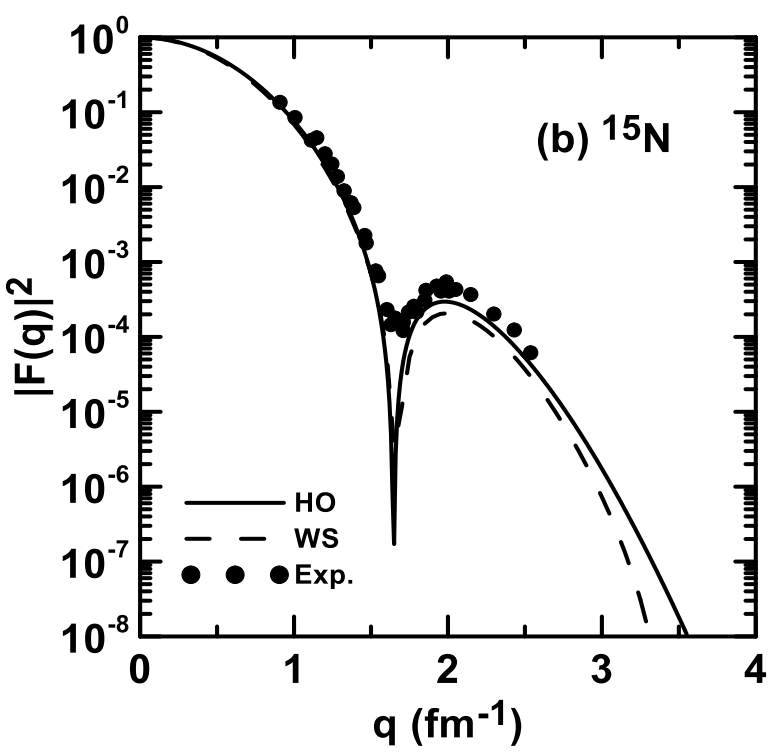

Fig.(3): The calculated form factors obtained by the WS and $\mathrm{HO}$ potentials for ${ }^{13} \mathrm{C}$ and ${ }^{15} \mathrm{~N}$ nuclei. The measured results (dotted symbols) for ${ }^{13} \mathrm{C}$ and ${ }^{15} \mathrm{~N}$ nuclei are taken from Refs. [19] and [22], respectively.

\section{Conclusions}

This study draws the following conclusions:

1. It is found that both the Wood-Saxson (WS) and harmonic oscillator (HO) potentials are capable of providing theoretical predictions on the structure of p-shell nuclei and be in a satisfactory description with those of measured data.

2. The quadrupole form factors contribution, which are calculated by undeformed $\mathrm{p}$ shell model, are given a good coincidence with the measured results for ${ }^{9} \mathrm{Be}$ and ${ }^{11} \mathrm{~B}$ nuclei.

\section{References}

[1] A. N. Antonov, P. E.Hodgson and I. Zh. Petkov, "Nucleon Momentum and Density Distribution in Nuclei", Clarendon Press, Oxford, (1988).
[2] R. Roy and B. P.Nigam, "Nuclear Physics Theory and Experiment", John and Sons, New York, (1967).

[3] D. J. Millener, D. I. Sober, H. Crannel, J. T. O'Brien, L. W. Fagg, S. Kowalski, C. F. Williamson and Lapikas, Physical Review C 39, 14, 1989.

[4] Gibson B. F., Goldberg A. and Weiss M. S., "Single-particle ground-state model of ${ }^{4}$ He" Nuclear Physics A 111, 321-330, 1968.

[5] Ridha A. R., "Study of charge density distributions, elastic charge form factors and root-mean square radii for ${ }^{4} \mathrm{He},{ }^{12} \mathrm{C}$ and ${ }^{16} \mathrm{O}$ nuclei using Woods- Saxon and harmonic-oscillator potentials" Iraqi Journal of Physics 14, 42-50, 2016. 
[6] Lojewski Z., Pomorska B. N., and Pomorski K., "Mean square radii of nuclei calculated with the Woods-Saxon potential" Physical Review C 51, 601-605, 1995.

[7] Gamba S., Ricco G. and Rottigni G., "A Phenomenological Woods-Saxon potential for p-shell nuclei" Nuclear Physics A 213, 383-396, 1973.

[8] Takeuchi K. and Moldauer P. A., "Neutron single particle levels in a WoodSaxon potential" Physics Letters 28B (1969) 384.

[9] Brown B. A., Massent S. E. and Hodgson P. E., "Proton and neutron density distributions for $A=16-58$ nuclei" J. Phys. G: Nucl. Phys. 5, 1655-1697, 1979.

[10] L. R. B. Etlon, "Nuclear sizes" Oxford University Press (1961).

[11] Stovall T., Goldemberg J. and Isabelle D. B., "Coulomb form factors of ${ }^{10} \mathrm{~B}$ and ${ }^{11}$ B" Nuclear Physics 86, 225-240, 1966.

[12] Suelzle L. R., Qearian M. R., and Rannell H. C., "Elastic electron scattering from ${ }^{6} \mathrm{Li}$ and ${ }^{7} \mathrm{Li}$ " Physical Review 162, 992-1005, 1967.

[13] A. Ozawa, T. Suzuki, I. Tanihata, "Nuclear size and related topics" Nuclear Physics A 693, 32-62, 2001.

[14] Vries H. D., Jager C.W., and Vries C., "Nuclear charge density distribution parameters from elastic electron scattering" Atomic data and nuclear data tables 36 (3), 495-536, 1987.

[15] Angeli I. and Marinova K.P., "Table of experimental nuclear ground state charge radii: An update" Atomic Data and Nuclear Data Tables 99, 69-95, 2013.

[16] Brown B. A., Etchegoyen A., Godwin N. S., Rae W. D. M., Richter W. A., Ormand W.E., Warburton E. K., Winfield J. S., Zhao L., Zimmerman C. H. 2005. Oxbash for Windows PC. MSU-NSCL report number 1289.

[17] Berkhout U. M., Ford K. W. and Green A. E. S., "Charge distributions of nuclei of the 1p-shell" Annals of Physics 8, 119-171, 1959.

[18] Schaller L. A., L., Schellenberg Phan T. Q., Piller G., Ruetschi A. and Schneuwly H., "Nuclear charge radii of the carbon isotopes ${ }^{12} \mathrm{C},{ }^{13} \mathrm{C}$ and ${ }^{14} \mathrm{C}$ " Nuclear Physics A 379, 523-535, 1982.
[19] Dally E. B., Croissiaux M. G. and Schweitz B., "Scattering of high-energy electrons by Nitrogen-14 and -15" Physical Review C 2, 2057- 2068, 1970.

[20] Jansen J. A., Peerdeman R. T. and de Vries C., "Nuclear charge radii of ${ }^{12} \mathrm{C}$ and ${ }^{9}$ Be" Nuclear Physics A 188, 337, 1972.

[21] Bernheim M., Stovall T. and Vinciguerra D., "Electron scattering from ${ }^{9} \mathrm{Be}$ " Nuclear Physics A 97, 488-504, 1967.

[22] Heisenberg J., Mccarthy J. S. and Sick I., "Elastic electron scattering from ${ }^{13} \mathrm{C}$ " Nuclear Physics A 157, 435-448, 1970. 